Supporting Information

\title{
Wavelength-Dependent Ultraviolet Photodissociation of Protonated Tryptamine
}

Kailin Zhang a,b, Lifu Ma ${ }^{a}$, Min Zhou ${ }^{b, c}$, Yingying Shi ${ }^{b}$, Shuqi Lib, Yan Wang *a, Xianglei Kong ${ }^{* b, d}$

a. School of Precision Instrument and Opto-Electronics Engineering,

TianjinUniversity, Tianjin 300072, China. E-mail:wangyan@tju.edu.cn

b. State Key Laboratory and Institute of Elemento-Organic Chemistry, Nankai University, Tianjin 300071, China. E-mail: kongxianglei@nankai.edu.cn

c. Department of Physics, Anhui Normal University, Wuhu 241000, China.

d. Collaborative Innovation Center of Chemical Science and Engineering, Nankai University, Tianjin 300071, China 
Scheme S1. $\beta$-carbon deuterated tryptamine.

\section{2-(1H-indol-3-yl)ethan-1,1-d 2 -1-amine Hydrochloride ${ }^{\text {Irefl }}$}<smiles>[2H]C([2H])(N)Cc1c[nH]c2ccccc12</smiles>

To a solution of samarium (II) iodide $(0.10 \mathrm{M}$ in THF, $12 \mathrm{~mL})$, a solution of 3-indoleacetonitrile $(0.20 \mathrm{mmol})$ in THF $(2.0 \mathrm{~mL})$ was added, followed by $\mathrm{Et}_{3} \mathrm{~N}(7.2$ mmol) and $\mathrm{D}_{2} \mathrm{O}(7.2 \mathrm{mmol})$ under $\mathrm{Ar}$ at room temperature and stirred vigorously. After $15 \mathrm{~min}$, excess $\mathrm{SmI}_{2}$ was oxidized by bubbling air through the reaction mixture. The reaction mixture was diluted with $\mathrm{CH}_{2} \mathrm{Cl}_{2}(10 \mathrm{~mL})$ and $\mathrm{NaOH}(10 \mathrm{~mL}, 1.0 \mathrm{M}$, aq). The aqueous layer was extracted with $\mathrm{CH}_{2} \mathrm{Cl}_{2}(3 \times 10 \mathrm{~mL})$. Organic layers were combined, washed with $\mathrm{Na}_{2} \mathrm{~S}_{2} \mathrm{O}_{3}(2 \times 20 \mathrm{~mL}$, sat, aq. $)$, dried over $\mathrm{MgSO}_{4}$, filtered and concentrated. The crude product was treated with a $3.0 \mathrm{M}$ solution of $\mathrm{HCl}$ in cyclopentylmethyl ether and filtered. The solid was then washed with diethyl ether and dried under vacuum to give $37.0 \mathrm{mg}$ of 2 aa in $93 \%$ yield as a white solid. ${ }^{1} \mathrm{H}$ NMR (300 MHz, DMSO- $\left.d_{6}\right) \delta 11.04(\mathrm{br}, 1 \mathrm{H}), 8.13(\mathrm{br}, 3 \mathrm{H}), 7.56(\mathrm{~m}, 1 \mathrm{H}), 7.36(\mathrm{~m}$, $1 \mathrm{H}), 7.23(\mathrm{~d}, J=2.1 \mathrm{~Hz}, 1 \mathrm{H}), 7.08(\mathrm{~m}, 1 \mathrm{H}), 6.99$ (m, 1H), 3.01 (s, 2H);HRMS (ESI-TOF) $\mathrm{m} / \mathrm{z} \mathrm{M}$ calcd for $\mathrm{C}_{10} \mathrm{H}_{11} \mathrm{D}_{2} \mathrm{~N}_{2}$ 163.1199, found $\mathrm{C}_{10} \mathrm{H}_{11} \mathrm{D}_{2} \mathrm{~N}_{2}$ 163.1198. Percentage of exchanged protons are determined by ${ }^{1} \mathrm{H}$ NMR and indicated in square brackets.

Ref : Ding, Y.; Luo, S.; Adijiang, A.; Zhao, H.; An, J. Reductive Deuteration of Nitriles: The Synthesis of $\alpha, \alpha$-Dideuterio Amines by Sodium-Mediated Electron Transfer Reactions. J. Org. Chem.2018, 83, 12269-12274. 
Scheme S2 McLafferty-type rearrangement<smiles>C=C1c2ccccc2NC1CN(C)CCc1c[nH]c2ccccc12</smiles> 
Fig. S1. The applied laser energies under different wavelengths in the experiment.

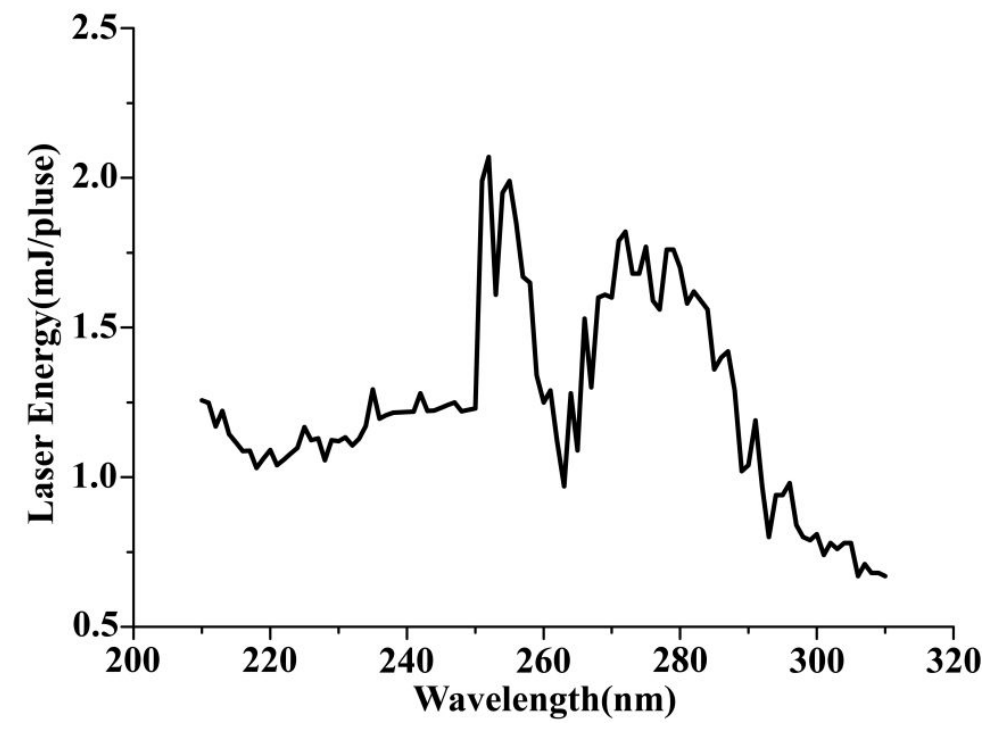


Fig. S2 Experimental UVPD spectra of $[\operatorname{Tryp}+\mathrm{H}]^{+}$under average energies at a) 1.4 $\mathrm{mJ} /$ pulse and b) $2.0 \mathrm{mj} /$ pulse.

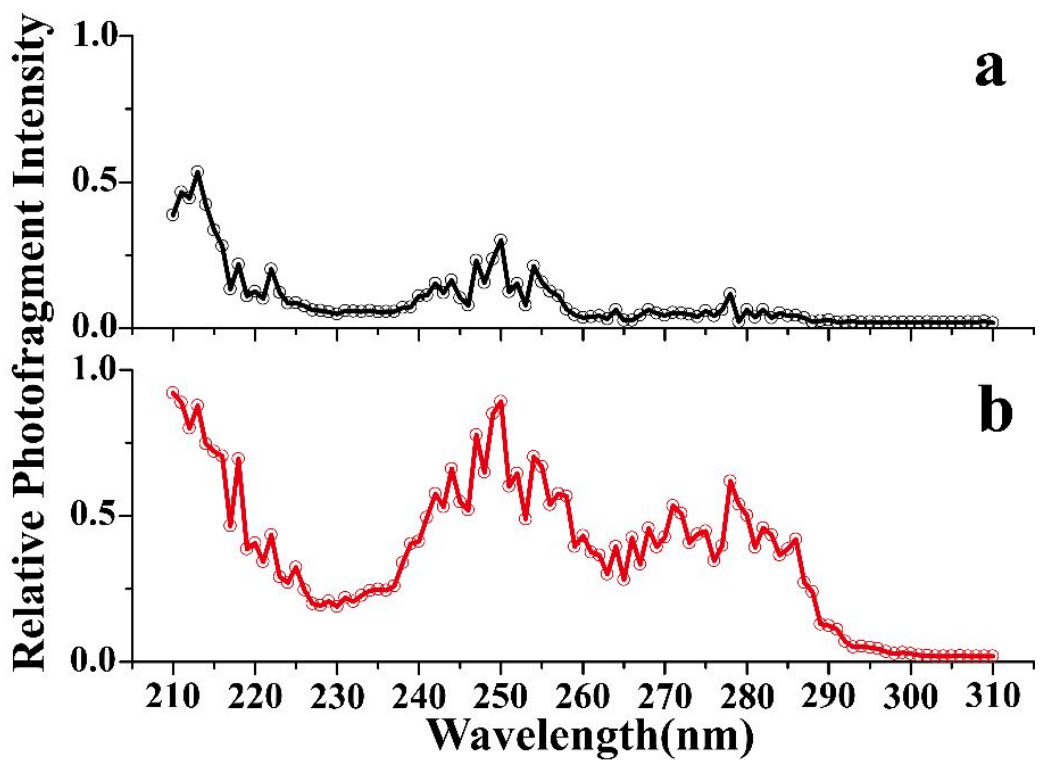


Figure S3 Experimental spectrum; a)-e) the yield UV spectra obtained of the five fragment ions at $\mathrm{m} / \mathrm{z} 143,130,128,117$ and 115, respectively. The experimental results are obtained in the range of $210-310 \mathrm{~nm}$.

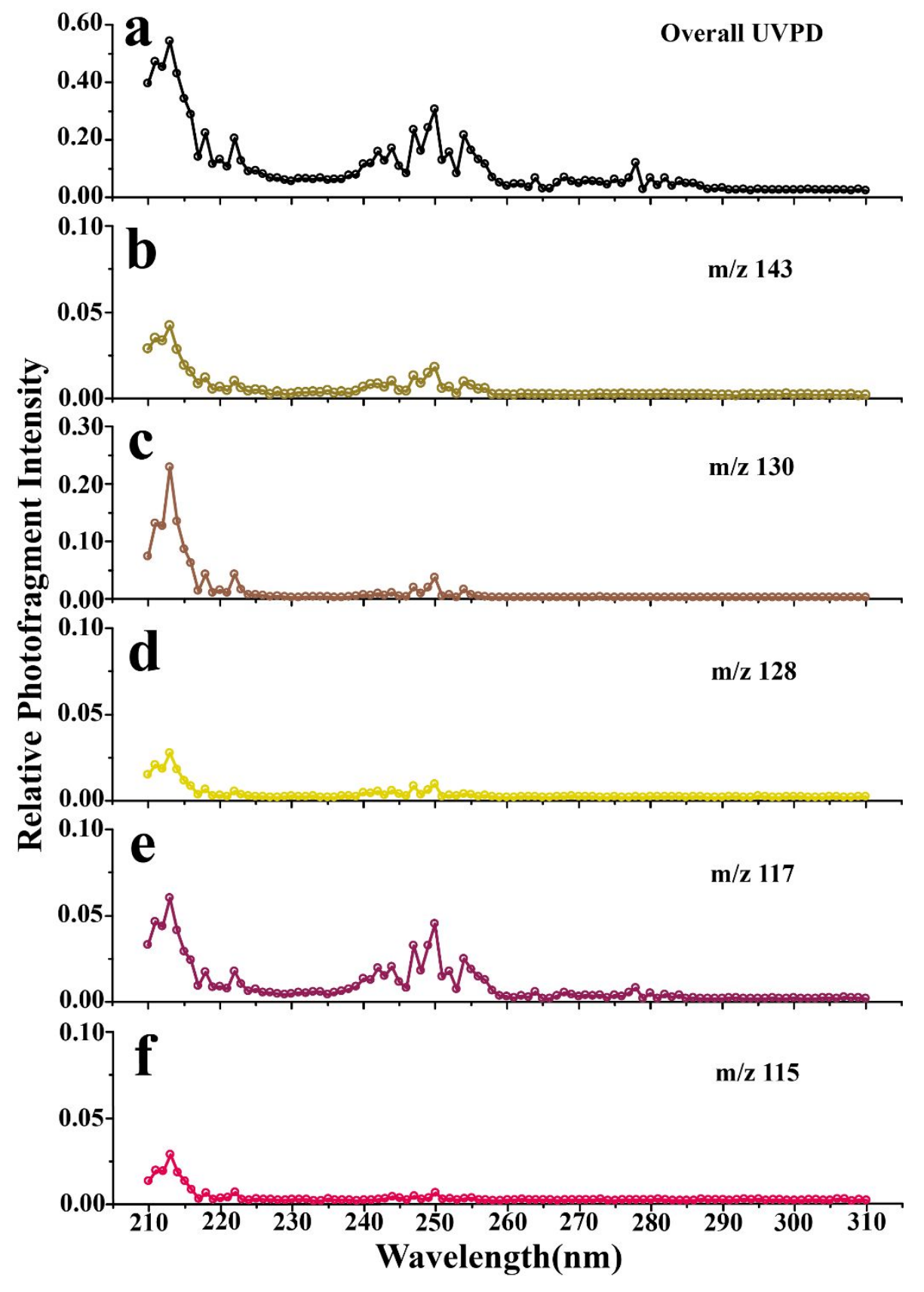


Figure S4 Structures of various $[\operatorname{Tryp}+\mathrm{H}]^{+}$isomers, including isomers with non-preferential protonation sites. Relative energies at PBE1PBE/6-311++g(d,p) level are given in $\mathrm{kcal} / \mathrm{mol}$.
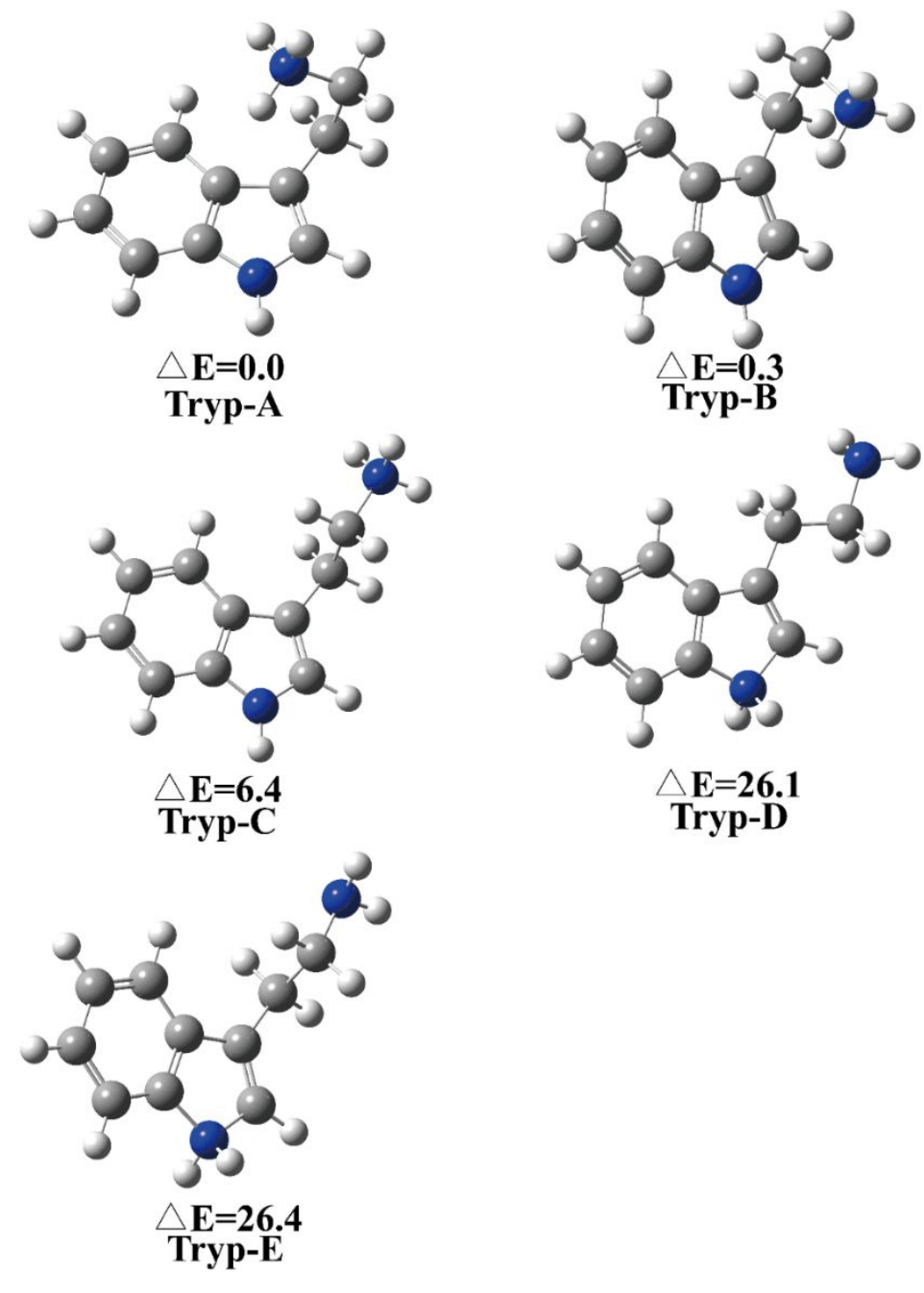

$$
\triangle \mathbf{E}=\mathbf{2 6 . 1}
$$

Tryp-D 
Figure. S5 a) Potential energy curves for dissociation of protonated tryptamine along the N-H dissociation coordinate obtained by DFT calculations for $\mathrm{S}_{0}$ and TDDFT calculations for $S_{1}, S_{3}$ and $S_{4}$ on the level of PBE0/6-311++g(d,p). b)Potential energy curves for dissociation of protonated tryptamine along the $\mathrm{C}-\mathrm{N}$ coordinates obtained by DFT calculations for $\mathrm{S}_{0}$ and TDDFT calculations for $\mathrm{S}_{1}$ and $\mathrm{S}_{3}\left(\mathrm{~S}_{4}\right)$ on PBE0/6-311++g(d,p) level. All the orbitals were drawn by Multiwfn 3.6.

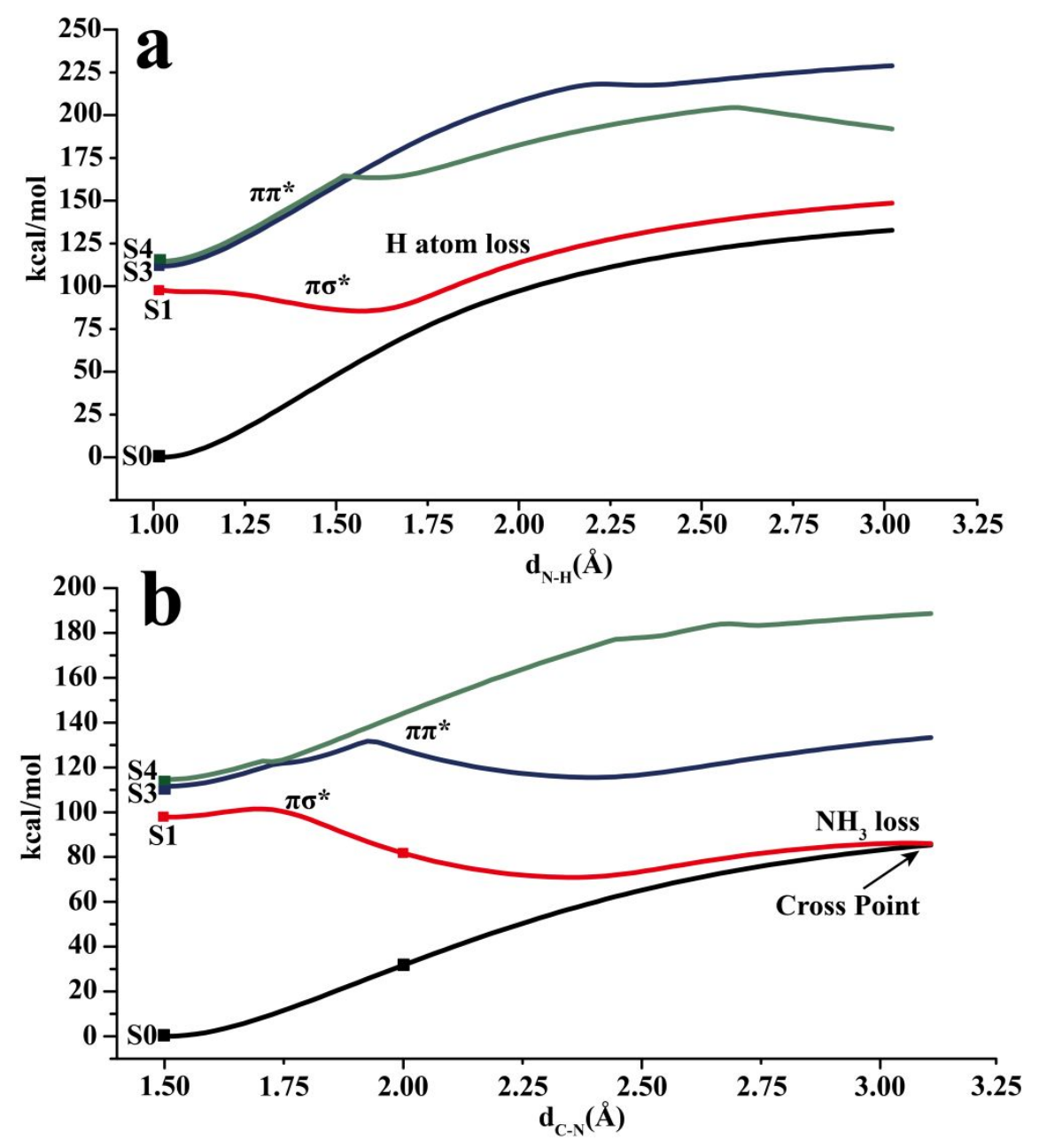


Figure S6 NPA analysis of $\mathrm{m} / \mathrm{z}=160$ and $\mathrm{m} / \mathrm{z}=144$ fragment ion calculated at PBE0/6-311++g(d,p) level. The charges are labeled beside each atom and marked by color. The green side stands for positive charge and the red side stands for negative charge.

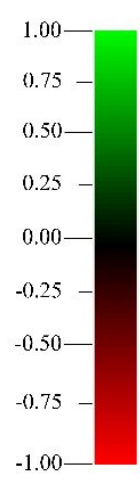

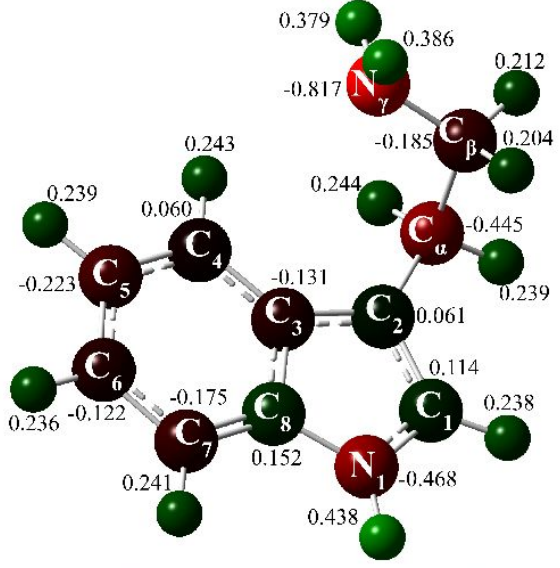

Fragment ion $\mathbf{m} / \mathbf{z}=160$

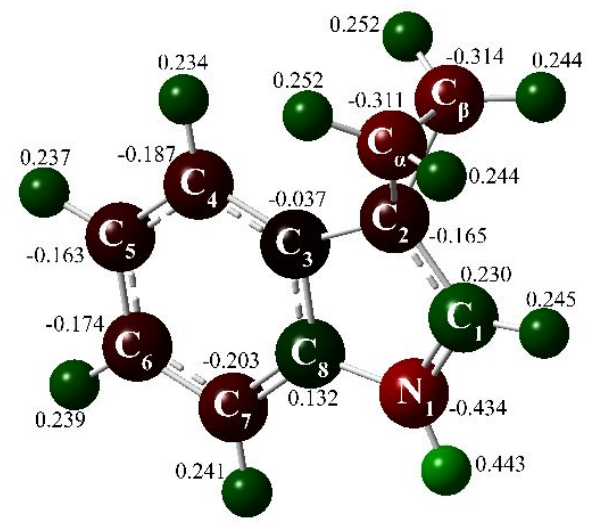

Fragment ion $m / z=144$ 
Figure S7 NPA analysis of each steps of MR reaction calculated at PBE0/6-311++g(d,p) level. The charges are labeled beside each atom and marked by color. The green side stands for positive charge and the red side stands for negative charge.
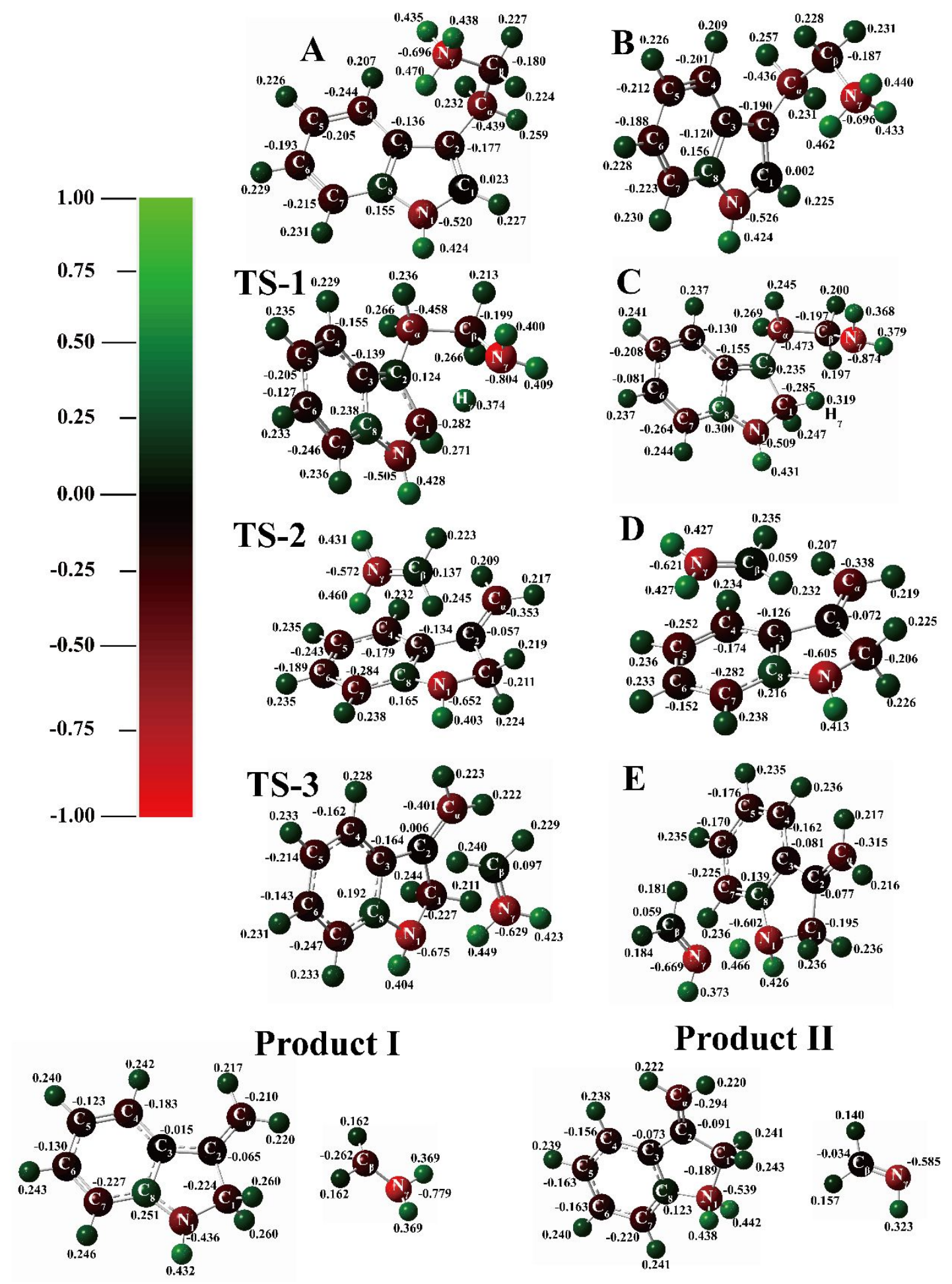
